\title{
Case report: Rhabdomyolysis in the setting of acute human immunodeficiency virus infection
}

\author{
Jason C. Huang ${ }^{1}$, Stephanie Gold ${ }^{2}$, Kathleen A. McManus ${ }^{1^{\star}}$, Brian Wispelwey ${ }^{3}$ \\ ${ }^{1}$ Department of Medicine, University of Virginia, Charlottesville, Virginia, USA \\ ${ }^{2}$ Department of Family Medicine, University of Colorado, Denver, Colorado, USA; ${ }^{*}$ Corresponding Author: Km8jr@virginia.edu \\ ${ }^{3}$ Division of Infectious Diseases and International Health, Department of Medicine, University of Virginia, Charlottesville, USA
}

Received 3 March 2013; revised 3 April 2013; accepted 1 May 2013

Copyright (c) 2013 Jason C. Huang et al. This is an open access article distributed under the Creative Commons Attribution License, which permits unrestricted use, distribution, and reproduction in any medium, provided the original work is properly cited.

\begin{abstract}
Acute HIV infection (AHI) is often marked by nonspecific and vague complaints, which make detection difficult. An 18-year-old man presented with one week history of subjective fevers with chills, nonproductive cough and generalized body aches. He was found to have rhabdomyolysis due to AHI. This report adds the most thoroughly investigated case of AHI-associated rhabdomyolysis to date, having ruled out erlichia, legionella, parainfluenza, adenovirus, enterovirus, metapneumovirus, RSV and DILS.
\end{abstract}

Keywords: Acute HIV Infection; Rhabdomyolysis; HIV; Primary HIV

\section{INTRODUCTION}

Acute HIV infection (AHI) represents the 2 to 8 week period between initial viral exposure and HIV antibody seroconversion [1]. Detection of AHI remains a significant dilemma and is often marked by a nonspecific influenza-like syndrome with malaise, myalgia, arthralgia, fever, pharyngitis and lymphadenopathy. Given the vague nature of this clinical presentation, $\mathrm{AHI}$ is a diagnosis that is often overlooked and missed. However, sometimes there is a unique presentation of AHI. We report the case of a young patient with primary HIV infection who presented with acute rhabdomyolysis.

\section{CASE REPORT}

An 18-year-old homosexual homeless male with no significant past medical history presented to the emergency department in the spring with a one week history of subjective fevers with chills, nonproductive cough and generalized body aches. He also reported a mild sore throat and dark urine for the previous few days. He denied nausea, vomiting, diarrhea, or any recent unexpected weight loss.

He reported multiple recent sick contacts and had not received a flu vaccination. The patient denied any significant physical activity preceding his symptoms, although he had been kicked in the hip approximately a week prior to presentation by an unknown assailant. While the patient admitted to intermittent marijuana use, he denied intravenous drug use and alcohol use. He had not recently traveled out of the country. Initially, on presentation, he reported that his last sexual encounter was 6 months before presentation and he reported that there was use of a condom. He reported one incidence of unprotected intercourse a year before presentation. During the patient's hospital admission, he admitted to three recent sexual partners with one partner within the month before presentation.

Vital signs on presentation were blood pressure 108/62, pulse 75 , temperature $37^{\circ} \mathrm{C}$, respiratory rate of 19 breaths/minute and oxygen saturation of $99 \%$ on room air. Physical examination revealed a well developed African American male in no acute distress. He did not have scleral icterus, cervical lymphadenopathy, organomegaly, or any significant rash. Marked tenderness to palpation was appreciated over the medial shins bilaterally. There was no tenderness to palpation or ecchymosis around the right hip where he reported being kicked.

Laboratory results included the following: white blood cell count, 2470/L (43\% neutrophils, 43\% lymphocytes, and $14 \%$ monocytes); hemoglobin, $17.1 \mathrm{~g} / \mathrm{dL}$; hematocrit, 47.9\%; platelets, 140,000/uL; sodium, $132 \mathrm{mEq} / \mathrm{dL}$; potassium, $4.1 \mathrm{mEq} / \mathrm{dL}$; chloride, $99 \mathrm{mEq} / \mathrm{dL}$; bicarbonate, $21 \mathrm{mEq} / \mathrm{dL}$; blood urea nitrogen, $20 \mathrm{mg} / \mathrm{dL}$; creatinine, $1.3 \mathrm{mg} / \mathrm{dL}$; calcium, $8.1 \mathrm{mg} / \mathrm{dL}$; aspartate aminotransferase, $1988 \mathrm{U} / \mathrm{L}$; alanine aminotransferase, $261 \mathrm{U} / \mathrm{L}$; 
thyroid stimulating hormone, $3.07 \mathrm{mIU} / \mathrm{mL}$; lactate dehydrogenase, $9964 \mathrm{U} / \mathrm{L}$; creatinine phosphokinase (CPK), 380,064 U/L. A urine specimen was brown and hazy, with moderate bilirubin, $3+$ protein, and moderate bacteria. Microscopy revealed 19 red blood cells, 15 white blood cells, 3 casts, and 7 epithelial cells. A urine drug screen was positive for cannabinoids only. Blood cultures were negative.

In subsequent laboratory evaluation, a combined assay for the HIV-1/HIV-2 Ab and p24 antigen test was reactive. Confirmatory Western blot for both HIV-1 and HIV-2 antibodies were negative, leading us to conclude that the patient was in acute seroconversion. HIV viral load was $>10,000,000$ copies. Serologies for hepatitis A, $\mathrm{B}$, and $\mathrm{C}$; Epstein-Barr virus (EBV); and ehrlichia were negative. Positive immunoglobulin $G$ antibodies were found for cytomegalovirus (CMV) but with a negative viral load. Urine legionella antigen was negative. Respiratory virus panel by polymerase chain reaction was negative for influenza A (H1 and $\mathrm{H} 3$ ) and B, parainfluenza 1 (1,2, and 3), metapneumovirus, rhinovirus/ enterovirus, adenovirus, and respiratory synctial virus (RSV) A and B. Blood and urine cultures were negative. This report adds the most thoroughly investigated case of AHI-associated rhabdomyolysis to date, having ruled out erlichia, legionella, parainfluenza, adenovirus, enterovirus, metapneumovirus, RSV and DILS.

Aggressive hydration was initiated with bicarbonate given the patient's significantly elevated CPK which remained persistently elevated $>300,000$ through his second hospital day despite aggressive intravenous fluid resuscitation. For the first few days after admission, he complained of weakness, leading to marked difficulty ambulating, which promptly resolved. On the fourth hospital day, a muscle biopsy was obtained to evaluate for diffuse lymphocytic infiltrative syndrome (DILS). The biopsy did not reveal any significant pathology. That same day he was also started on highly active anti-retroviral therapy (HAART) with a combination pill of emtricitabine, tenofovir, and efavirenz. His neutropenia, thrombocytopenia, and acute kidney injury resolved and he was discharged on hospital day 10 with marked improvement in his CPK to less than 10,000. Unfortunately, the patient was lost to follow-up despite multiple attempts to establish care for him, in both the primary care and infectious disease clinics, following this hospital discharge.

\section{DISCUSSION}

From a public health perspective, there continues to be growing evidence that improved identification of AHI is essential to the management of HIV. Fiebig et al., described six stages of AHI indicating a 10 day span between the ability to detect HIV RNA before enzyme immunoassays (EIAs) are able to detect HIV antibodies [2]. This 10 day period is split by the ability to detect the p24 antigen, whereas Western blot testing lags behind the EIA test by 1 week to 1 month. Fourth generation EIAs, such as the one used with this patient, enhance sensitivity for AHI detection by combined antigen-antibody testing as a screen for the p24 antigen and HIV antibodies [1].

Multiple prior studies have shown evidence that AHI represents a time period with increased risk for sexual transmission of the virus. Pilcher et al., found a correlation between HIV concentration in blood and semen [3]. More importantly, there appeared to be a clear peak in viral load within the first 2 months of acute infection in comparison to the viral load during the year following initial exposure. In conjunction, Wawer et al., retrospectively illustrated the highest rates of HIV transmission amongst 235 Ugandan couples in the early stage of HIV infection [4]. Powers et al., developed a mathematical model describing HIV transmission in Lilongwe, Malawi [5]. Based upon clinic data over an 18 year period, 38\% of heterosexual HIV transmission was attributable to individuals with early infection.

Yet, opportunities for screening for AHI are missed in the outpatient and inpatient settings [6,7]. The nonspecific influenza-like syndrome of AHI is easily overlooked. A detailed social history is paramount, but as illustrated in our case report, patients are often not forthcoming with certain risk factors such as unsafe sexual practice during initial discussions with healthcare teams.

Rhabdomyolysis is a syndrome marked by skeletal muscle damage leading to the release of intracellular products into the bloodstream. Muscle cell lysis leads to the release of myoglobin, CPK as well as intracellular electrolytes. Thus, the laboratory findings of rhabdomyolysis are often marked by electrolyte disarray with hyperkalemia, hyperphosphatemia and an associated hypocalcemia due to calcium phosphate deposition in the peripheral tissue.

The primary causes for rhabdomyolysis include trauma, significant physical exertion, sustained hypoxia, electrolyte disarray, drugs/toxins as well as certain infections [8]. A review by Singh and Scheld in 1996 reviewed the published case reports of rhabdomyolysis attributed to viral and bacterial infection [9]. Fifty-nine cases of viral-associated rhabdomyolysis were found, with influenza (25 cases), HIV (8 cases) and Coxsackie virus (8 cases) representing the most common causes. The remaining cases were caused by EBV, CMV, echovirus, adenovirus, herpes simplex, parainfluenza and varicella-zoster virus [9].

Rhabdomyolysis has more commonly been associated with HIV infection beyond the period of acute serocon- 
version. Neves et al., described rhabdomyolysis in a patient with chronic HIV, while Younger et al. described a patient with recurrent myoglobinuria due to polymyositis $[10,11]$. Multiple cases of rhabdomyolysis were noted in two separate case series by Chariot et al. and Joshi/Liu $[12,13]$. The true etiology underlying each patient's presentation was complicated, however, by an array of factors such as concomitant use of alcohol and illicit drugs, bacterial infection and alternative iatrogenic sources. In addition, zidovudine, an integral medication to HIV therapy for many individuals, is one of the multiple medications associated with drug-induced rhabdomyolysis [14].

Twelve total published cases of rhabdomyolysis have been associated with acute seroconversion of HIV and briefly summarized in Table 1 [15-27]. All of these cases documented acute HIV seroconversion and ruled out most of the other potential contributing factors including sources of infection (blood/urine cultures), electrolyte disarray, illicit drug use, history of alcohol use and serologies for influenza, CMV, EBV, Hepatitis $\mathrm{A} / \mathrm{B} / \mathrm{C}$, and toxoplasmosis.

Acute kidney injury (AKI) is a commonly associated complication of rhabdomyolysis. The pathophysiology is attributed to a combination of vasoconstriction, direct tubular injury and obstruction instigated by an elevated serum myoglobin that is excreted into the urine [28]. Much of the nephrotoxic effects of myoglobin occur with acidic urine output, which leads to myoglobin crystallization and free radical formation. In a study by Melli et al., a retrospective review of patient charts at one hospital from 1993 to 2001 revealed 475 patients with rhabdomyolysis [14]. Of these patients, 218 (46\%) were found to suffer from AKI. The review by Singh and Scheld revealed that 11 of the 25 patients (44\%) with influenza-associated rhabdomyolysis were associated with AKI [9]. In comparison, the compilation of patients presenting with rhabdomyolysis in the setting of AHI also follows a similar pattern with 6 of the 12 patients listed above with known serum creatinine values exhibiting AKI upon presentation.

\section{CONCLUSION}

AHI continues to represent a diagnostic dilemma in the medical field. In 2006, the Centers for Disease Control and Prevention released guidelines advocating for routine screening of all patients aged 13 - 64 years of age regardless of risk factors [29]. There continues to be significant debate over the optimal way of enhancing the detection of AHI [30-32]. Regardless, recognition of the unique presentations of AHI remains useful in the acute

Table 1. Cases of rhabdomyolysis attributed to acute HIV infection.

\begin{tabular}{|c|c|c|c|c|c|c|}
\hline Reference & Age & Sex & Presenting symptoms & $\begin{array}{l}\text { CPK peak } \\
\quad(\mathbf{U} / \mathbf{L})\end{array}$ & AKI & Additional or complicating features \\
\hline Mahe et al., 1989 [15] & 18 & M & Myalgias, sore throat & 5750 & & No alcohol history or toxicology screen \\
\hline del Rio et al., 1990 [16] & 29 & M & Fever, sore throat & 4224 & Yes & No toxicology screen \\
\hline Guillaume et al., 1995 [17] & 42 & M & Fever, myalgias & 63,800 & $\begin{array}{l}\text { Yes } \\
(\mathrm{Cr} 1.9)\end{array}$ & Myopericarditis \\
\hline Rastegar et al., 2001 [18] & 51 & M & Lower extremity (LE) pain & 32,720 & $\begin{array}{c}\text { Yes } \\
(\mathrm{Cr} 1.6)\end{array}$ & $\begin{array}{l}\text { History of alcohol use without alcohol } \\
\text { level, no CMV or EBV serologies }\end{array}$ \\
\hline McDonagh et al., 2003 [19] & 33 & M & Fever, sore throat, LE pain & 18,840 & No & \\
\hline Delo et al., 2006 [20] & 46 & & Fever, cough, rash & 5638 & & \\
\hline Tattevin, 2007 [21] & 16 & $\mathrm{~F}$ & $\begin{array}{l}\text { Fever, myalgias, diarrhea, } \\
\text { lymphadenopathy }\end{array}$ & 2734 & No & Notable transaminitis AST 4266, ALT 1875 \\
\hline Prabahar et al., 2008 [23] & 42 & M & $\begin{array}{l}\text { LE pain, vomiting, } \\
\text { SOB, dark urine }\end{array}$ & 278,000 & $\begin{array}{c}\text { Yes } \\
(\text { Cr 6.8) }\end{array}$ & $\begin{array}{l}\text { LE edema, lymphadenopathy, notable } \\
\text { transaminitis AST 3640, ALT } 419\end{array}$ \\
\hline Maniar et al., 2009 [24] & 16 & M & Fever, diarrhea, myalgias & 203,375 & No & Pediatric age \\
\hline Douvoyiannis et al., 2009 [25] & 20 & M & Fever, diarrhea, sore throat & 2968 & $\begin{array}{c}\text { Yes } \\
(\mathrm{Cr} 1.6)\end{array}$ & Concurrent encephalopathy \\
\hline Pano-Pardo et al., 2009 [26] & 19 & $\mathrm{~F}$ & Weakness, LE pain, rash & 10,681 & $\begin{array}{l}\text { Yes } \\
(\mathrm{Cr} 2.3)\end{array}$ & $\begin{array}{l}\text { Myocarditis, pancreatitis, AKI with bilateral } \\
\text { renal infarcts on CT; CMV not ruled out }\end{array}$ \\
\hline Takahashi 2011 [27] & 21 & M & Altered mental status & 218,100 & No & Written in Japanese \\
\hline Huang et al. 2013 & 18 & M & Fever, myalgias, sore throat & 380,064 & $\begin{array}{c}\text { Yes } \\
(\mathrm{Cr} 1.3)\end{array}$ & $\begin{array}{l}\text { Preceding trauma to the hip one week prior } \\
\text { to presentation, but no subsequent } \\
\text { hematoma or limitation to range of motion }\end{array}$ \\
\hline
\end{tabular}


care setting. Our patient presented to the Emergency Department with fever, myalgia and was found to have a serum CPK higher than any prior patient with AHI-associated rhabdomyolysis.

\section{REFERENCES}

[1] Cohen, M.S., Gay, C.L., Busch, M.P. and Hecht, F.M. (2010) The detection of acute HIV infection. Journal of Infectious Disease, 202, S270-S277. doi:10.1086/655651

[2] Fiebig, E.W., Wright, D.J., Rawal, B.D., et al. (2003) Dynamics of HIV viremia and antibody seroconversion in plasma donors: Implications for diagnosis and staging of primary HIV infection. AIDS, 17, 1871-1879. doi:10.1097/00002030-200309050-00005

[3] Pilcher, C.D., Tien, H.C., Eron Jr., J.J., et al. (2004) Brief but efficient: Acute HIV infection and the sexual transmission of HIV. Journal of Infectious Disease, 189, 17851792. doi:10.1086/386333

[4] Wawer, M.J., Gray, R.H., Sewankambo, N.K., et al. (2005) Rates of HIV-1 transmission per coital act, by stage of HIV-1 infection, in Rakai, Uganda. Journal of Infectious Disease, 191, 1403-1409. doi:10.1086/429411

[5] Powers KA, Ghani AC, Miller WC, et al. (2011) The role of acute and early HIV infection in the spread of HIV and implications for transmission prevention strategies in liLongwe, Malawi: A modelling study. Lancet, 378, 256268. doi:10.1016/S0140-6736(11)60842-8

[6] Liddicoat, R.V., Horton, N.J., Urban, R., Maier, E., Christiansen, D. and Samet, J.H. (2004) Assessing missed opportunities for HIV testing in medical settings. Journal of General Internal Medicine, 19, 349-356. doi:10.1111/j.1525-1497.2004.21251.x

[7] Weintrob, A.C., Giner, J., Menezes, P., et al. (2003) Infrequent diagnosis of primary human immunodeficiency virus infection: Missed opportunities in acute care settings. Archives of Internal Medicine, 163, 2097-2100. doi:10.1001/archinte.163.17.2097

[8] Allison, R.C. and Bedsole, D.L. (2003) The other medical causes of rhabdomyolysis. American Journal of Medical Science, 326, 79-88.

doi:10.1097/00000441-200308000-00005

[9] Singh, U. and Scheld, W.M. (1996) Infectious etiologies of rhabdomyolysis: Three case reports and review. Clinical Infectious Diseases, 22, 642-649. doi:10.1093/clinids/22.4.642

[10] Neves, O., Stein, C.M., Thornton, C., Gangaidzo, I. and Thomas, J.E. (1991) Rhabdomyolysis associated with human immunodeficiency virus (HIV) infection. Central African Journal of Medicine, 37, 387-388.

[11] Younger, D.S., Hays, A.P., Uncini, A., Lange, D.J., Lovelace, R.E. and DiMauro, S. (1989) Recurrent myoglobinuria and HIV seropositivity: Incidental or pathogenic association? Muscle and Nerve, 12, 842-843. doi:10.1002/mus.880121009

[12] Chariot, P., Ruet, E., Authier, F.J., Levy, Y. and Gherardi, R. (1994) Acute rhabdomyolysis in patients infected by human immunodeficiency virus. Neurology, 44, 1692-
1696. doi:10.1212/WNL.44.9.1692

[13] Joshi, M.K. and Liu, H.H. (2000) Acute rhabdomyolysis and renal failure in HIV-infected patients: Risk factors, presentation, and pathophysiology. AIDS Patient Care STDS, 14, 541-548. doi:10.1089/108729100750018308

[14] Melli, G., Chaudhry, V. and Cornblath, D.R. (2005) Rhabdomyolysis: An evaluation of 475 hospitalized patients. Medicine (Baltimore), 84, 377-385. doi:10.1097/01.md.0000188565.48918.41

[15] Mahe, A., Bruet, A., Chabin, E. and Fendler, J.P. (1989) Acute rhabdomyolysis coincident with primary HIV-1 infection. Lancet, 2, 1454-1455. doi:10.1016/S0140-6736(89)92061-8

[16] del Rio, C., Soffer, O., Widell, J.L., Judd, R.L. and Slade, B.A. (1990) Acute human immunodeficiency virus infection temporally associated with rhabdomyolysis, acute renal failure, and nephrosis. Reviews of Infectious Diseases, 12, 282-285. doi:10.1093/clinids/12.2.282

[17] Guillaume, M.P., Van Beers, D., Delforge, M.L., Devriendt, J. and Cogan, E. (1995) Primary human immunodeficiency virus infection presenting as myopericarditis and rhabdomyolysis. Clinical Infectious Diseases, 21, 451-452. doi:10.1093/clinids/21.2.451

[18] Rastegar, D., Claiborne, C., Fleisher, A. Matsumoto, A. (2001) A patient with primary human immunodeficiency virus infection who presented with acute rhabdomyolysis. Clinical Infectious Diseases, 32, 502-504. doi:10.1086/318508

[19] McDonagh, C.A. and Holman, R.P. (2003) Primary human immunodeficiency virus type 1 infection in a patient with acute rhabdomyolysis. Southern Medical Journal, 96, 1027-1030. doi:10.1097/01.SMJ.0000077066.27020.9C

[20] Delo, D., Brett, A.S. and Postic, B. (2006) Primary HIV infection presenting with acute rhabdomyolysis. American Journal of Medical Sciences, 332, 46-47. doi:10.1097/00000441-200607000-00010

[21] Tattevin, P., Camus, C., Arvieux, C., Ruffault, A. and Michelet, C. (2007) Multiple organ failure during primary HIV infection. Clinical Infectious Diseases, 44, e28-e29. doi:10.1086/510683

[22] Nicolas, X., Granier, H., Le Guen, P. and Talarmin, F. (2007) Acute rhabdomyolysis and primary human immunodeficiency virus type 1 infection: A new observation. La Revue de Médecine Interne, 28, 322-325. doi:10.1016/j.revmed.2007.01.008

[23] Prabahar, M.R., Jain, M., Chandrasekaran, V., Indhumathi, E. and Soundararajan, P. (2008) Primary HIV infection presenting as non-traumatic rhabdomyolysis with acute renal failure. Saudi Journal of Kidney Diseases and Transplantation, 19, 636-642.

[24] Maniar, A.J. and Tolan, R.W. (2009) Rhabdomyolysis as the presenting manifestation of acute human immunodeficiency virus infection in an adolescent. Infectious Diseases in Clinical Practice, 17, 12-16. doi:10.1097/IPC.0b013e318184d767

[25] Douvoyiannis, M. and Litman, N. (2009) Acute encepha- 
lopathy and multi-organ involvement with rhabdomyolysis during primary HIV infection. International Journal of Infectious Diseases, 13, e299-e304. doi:10.1016/j.ijid.2009.01.005

[26] Pano-Pardo, J.R., Alcaide, M.L., Abbo, L and Dickinson, G. (2009) Primary HIV infection with multisystemic presentation. International Journal of Infectious Diseases, 13, e177-e180. doi:10.1016/j.ijid.2008.09.010

[27] Takahashi, T. (2011) A case of primary human immunodeficiency virus infection with severe rhabdomyolysis without acute renal failure. Kansenshogaku Zasshi, 85, 268-271.

[28] Bosch, X., Poch, E. and Grau, J.M. (2009) Rhabdomyolysis and acute kidney injury. The New England Journal of Medicine, 361, 62-72. doi:10.1056/NEJMra0801327

[29] Branson, B.M., Handsfield, H.H., Lampe, M.A., et al. (2006) Revised recommendations for HIV testing of adults, adolescents, and pregnant women in health-care settings. MMWR Recommendations and Reports, 55, 117.

[30] Cunningham, C.O., Doran, B., DeLuca, J., Dyksterhouse, R., Asgary, R. and Sacajiu, G. (2009) Routine opt-out HIV testing in an urban community health center. AIDS Patient Care and STDS, 23, 619-623. doi:10.1089/apc.2009.0005

[31] Bartlett, J.G., Branson, B.M., Fenton, K., Hauschild, B.C., Miller, V. and Mayer, K.H. (2008) Opt-out testing for human immunodeficiency virus in the united states: Progress and challenges. The Journal of the American Medical Association, 300, 945-951. doi:10.1001/jama.300.8.945

[32] Kerndt, P.R., Dubrow, R., Aynalem, G., et al. (2009) Strategies used in the detection of acute/early HIV infections. The NIMH multisite acute HIV infection study: I. AIDS and Behavior, 13, 1037-1045. doi:10.1007/s10461-009-9580-8 